\title{
On the choice of control objects in experimental gerontological research
}

\author{
A.N. Khokhlov*, A.A. Klebanov, G.V. Morgunova \\ School of Biology, Lomonosov Moscow State University, Moscow, Russia \\ *e-mail:khokhlov@mail.bio.msu.ru
}

Key words: aging, senescence, geroprotectors, control groups, cell cultures, experimental animals

Recently, a large number of papers have appeared that describe the successful use of various biologically active compounds (mitochondrial antioxidants, antidiabetic biguanides, mimetics of dietary restriction, etc.) as geroprotectors. However, in our opinion, in most cases, the positive results of such studies are determined by a "successful" selection of control objects. As such, animals with some abnormalities are often used $[1,2]$, so that any favorable effect on the corresponding pathological processes leads to an increase in life span. Besides, control animals can be normal, i.e. wild type, but placed in some extreme conditions, which can be overcome precisely by certain biologically active compounds. Thus, treatment of pathologies is present, and not an effect on the fundamental processes of aging [3]. There is a point of view, according to which the results of Clive McKay's experiments, which have significantly prolonged the life of rats by limiting caloric intake, are determined, firstly, by the fact that the control animals fed ad libitum (which is not at all characteristic of animals in the wild), and secondly, because the Fisher-344 rats used in experiments are short-lived. It should be said that the above considerations seem to concern also gerontological experiments on cultured cells. In particular, we sometimes hear from our colleagues remarks about the model of stationary phase aging of cell cultures used in our laboratory due to the fact that most of the experiments are carried out on transformed rather than normal cells. However, this approach seems to us quite justified, because the phenomenon of stationary phase/chronological aging is common to a wide variety of cells, including bacteria, yeasts, cyanobacteria, mycoplasmas, animal and plant cells [4]. Cells with unlimited mitotic potential do not change either from experiment to experiment or during long-term cultivation without subcultivation [3], which cannot be said of normal diploid fibroblasts, whose telomeres are shortened with each division (and from the moment of seeding of the cells at low density to their entering the stationary phase of growth they can divide up to 10 times!).

Conclusion: To search for effective geroprotectors, which provide an impact on the fundamental mechanisms of aging, it is necessary to conduct studies on "maximally healthy" animals or on "maximally stable" model systems.

\section{References}

1. Shabalina I.G. et al. (2017) Improved health-span and lifespan in mtDNA mutator mice treated with the mitochondrially targeted antioxidant SkQ1. Aging (Albany NY). 9(2):315-336.

2. Takeda T., Hosokawa M., Higuchi K. (1997) Senescence-accelerated mouse (SAM): a novel murine model of senescence. Exp. Gerontol. 32(1-2):105-109.

3. Khokhlov A.N., Klebanov A.A., Morgunova G.V. (2018) Anti-aging drug discovery in experimental gerontological studies: from organism to cell and back. In: Aging: exploring a complex phenomenon, Ed. by Sh.I. Ahmad, Boca Raton: Taylor \& Francis. 577-595.

4. Morgunova G.V., Klebanov A.A., Khokhlov A.N. (2016) Some remarks on the relationship between autophagy, cell aging, and cell proliferation restriction. Moscow Univ. Biol. Sci. Bull. 71(4):207-211. 